\title{
Polarized Deuteron Source for Van de Graaff Accelerator
}

\author{
Yu.A. Plis" ${ }^{*}$, J. Černý ${ }^{b}$, A.N. Fedorov ${ }^{a}$, I.V. Gapienko ${ }^{a}$, G.M. Gurevich ${ }^{a, c}$, Z. Kohout ${ }^{b}$, \\ J. Petrík ${ }^{b}$, S. Pospíšil ${ }^{b}$, M. Solar ${ }^{b}$, J. Šveida $^{b}$, Yu.A. Usov $^{a}$, I. Wilhelm ${ }^{b}$ \\ a Joint Institute for Nuclear Research \\ Joliot-Curie, 6, 141980 Dubna, Moscow Region, Russia \\ ${ }^{b}$ Czech Technical University in Prague, Institute for Experimental and Applied Physics, \\ Horska, 3a/22, 12800 Prague 2, Czech Republic \\ ${ }^{c}$ Institute for Nuclear Research, Russian Academy of Science \\ Prospekt 60-letiya Oktyabrya, 7a, 117312 Moscow, Russia \\ E-mail: plisejinr.ru
}

\begin{abstract}
An attempt is made to develop a polarized deuteron source suited for the Van de Graaff accelerator of Czech Technical University in Prague. We base on Kaminsky's experiment on channeling deuterons through a Ni single crystal. The setup is described, which contains permanent magnets with a transversal magnetic field to increase the deuteron polarization using the Sona method (zero transition). The measurements of tensor polarization were carried out with a TiT target. The result is $P_{z z}=-0.12 \pm 0.04$ for a weak field at the target without channeling. The ultimate aim is to produce $14-\mathrm{MeV}$ polarized neutrons which will be used jointly with the frozen-spin polarized deuteron target for measurement of $\Delta \sigma_{T}$ and $\Delta \sigma_{L}$ asymmetries in the $n d$-transmission experiment.
\end{abstract}

PACS: $29.25 \mathrm{Lg} ; 29.25 \mathrm{Dz}$

23rd International Spin Physics Symposium - SPIN2018 -

10-14 September, 2018

Ferrara, Italy

${ }^{*}$ Speaker. 


\section{Introduction}

Formally, measurement of spin-dependent parameters allows imposing additional constraints on the reaction mechanism and the structure of the micro-object under study. For example, measurements of $\Delta \sigma_{T}$ and $\Delta \sigma_{L}$ are necessary to determine the imaginary part of the $n d$ forward elastic scattering amplitude [1]. These measurements are important for the problem of 3-nucleon forces [2].

The total cross-section difference $\left(\Delta \sigma_{L}\right)_{d}$ was measured at TUNL (North Carolina) [3] for the incident neutron energies of 5.0, 6.9, and $12.3 \mathrm{MeV}$.

In the previous experiments on $n p$-scattering (Prague, Charles University) [4, 5], transversely polarized neutrons were produced as a secondary beam in the $\mathrm{T}(d, n)^{4} \mathrm{He}$ reaction with deuterons with energies up to $2.5 \mathrm{MeV}$ [6]. The neutron beam with an energy $E_{n}=16.2 \mathrm{MeV}$ was emitted at an angle $\theta_{\text {lab }}=62.0^{\circ}$. The neutron polarization was $P_{n}=-0.135 \pm 0.014$. To get longitudinal beam polarization in the $\left(\Delta \sigma_{L}\right)_{p}$ experiment, the neutron spin was rotated with the help of a permanent magnet of $0.5 \mathrm{~T} \mathrm{~m}$. The associated particle method was used in the experiment.

The planned experiments are continuation in the Czech Technical University in Prague of the previous measurements of the same quantities in $n p$-scattering.

Preliminary experiments showed that the polarization and intensity of the neutron beam and also the deuteron polarization of the target are insufficient for achieving the necessary accuracy of the measurement of the cross-section difference [7]. To improve the parameters of the neutron beam, it is proposed to use the reaction $\mathrm{T}(d, n)^{4} \mathrm{He}$ with polarized deuterons with an energy of 100$150 \mathrm{keV}$. Also, we plan to replace the current target material (propanediol) with a novel material chemically doped with the trityl family radicals, which showed the deuteron polarization as high as $P_{d}=0.80$ [8].

As to the polarized beam, the first proposal concerning the nuclear polarization via a pick-up of polarized ferromagnetic electrons was made by Zavoiskii in 1957 [9]. The method proposes adiabatic transition of atoms from a high magnetic field to a low magnetic field on the order of 1 $\mathrm{mT}$ where nuclei are polarized through the hyperfine interaction.

Here we rely on the results of the experiment by Kaminsky $[10,11]$ on the production of nuclear polarized deuterium atoms by channeling a low energy deuteron beam through a magnetized single-crystal nickel foil.

In Kaminsky's setup a beam of deuterons with a half angle of $0.01^{\circ}$ was incident on a $\mathrm{Ni}(110)$ foil $\approx 2 \mu \mathrm{m}$ thick within $0.1^{\circ}$ of the [110] direction (the critical acceptance angle $(1.6-1.8)^{\circ}$ ). This is a hyperchanneling regime. He obtained $500 \mathrm{nA} / \mathrm{cm}^{2}$ of channeled deuterium atoms with an energy of 100-200 keV with nuclear polarization $P_{z z}=-0.32 \pm 0.010$ (without a significant lattice damage for $25 \mathrm{~h}$ of operating time). To test Zavoiskii's proposal, the author passed deuterons through magnetized polycrystalline foils and observed no significant tensor polarization (i.e. $P_{z z} \approx$ $0)$.

Feldman et al. [12] also made polarization measurements with an experimental arrangement very similar to that of Kaminsky. Their data qualitatively agree with Kaminsky's data $\left(P_{z z}=\right.$ $-0.14 \pm 0.06)$. Also, as in Kaminsky's experiment, no effect was seen for polycrystalline foils.

Quite a different electron field-emission experiment [13] on Ni showed that electrons emitted along the [100], [110], and [137] directions had predominantly a spin-up (along the magnetic field), 
but when emitted along the [111] direction, they had a spin-down .

Rau and Sizmann [14], who also used the ${ }^{3} \mathrm{H}(\mathrm{d}, \mathrm{n})^{4} \mathrm{He}$ reaction, measured polarization of nuclei in neutral deuterium atoms created by electron capture during reflection of a $150-\mathrm{keV} \mathrm{D}^{+}$beam incident at glancing angles $\left(<0.4^{\circ}\right)$ on the surface of magnetized $\mathrm{Ni}$ crystals.

The results show that the electron spin orientation is predominantly parallel to the magnetizing field for electrons in the (100), (110), and (111) surfaces and antiparallel in the (120) surface. In the (110) surface the electron polarization is $P=96 \%$ [15]. This explains the high polarization in Kaminsky's experiment.

On the other hand, there is evidence for polarization of $1 s$ electrons $\left(P_{1 s}=0.10 \pm 0.03\right)$ attached to F ions as they emerge from magnetized polycrystalline Fe layers [16].

\section{Theory}

Ebel [17] tried to explain the high observed polarization by postulating that once a deuteron captured a spin-up electron inside the crystal, the probability of its losing this electron would be small since the spin-up 3d-band states are filled. A captured spin-down electron, on the other hand, could readily be lost since the spin-down 3d-band states in the crystal are not filled. This would give rise to a pumping of electrons from spin-down to spin-up atomic states of deuterium.

Brandt and Sizmann [18], however, pointed out that stable bound electronic states could not exist in deuterium atoms passing through metals at these velocities. They proposed instead that the electron capture took place in the tail of the electron density distribution at the crystal surface where the density was low enough for bound states to be stable.

Later, Kreussler and Sizmann [19] discussed that at high energies (more than $250 \mathrm{keV} / \mathrm{amu}$ ) neutralization took place chiefly in the bulk of the crystal, and the surface effects were important at lower energies.

Kaminsky did not measure the polarization of nonchanneled atoms. We may suppose that the channeling is not essential, and the electron polarization in the neutral beam is determined by the polarization of the electrons at the surface.

\section{Experimental setup}

The scheme of the experimental setup is shown in Fig.1. We propose to apply the Sona method, zero-field transitions with total transfer of the electron polarization to deuterons in the atomic beam [20]. We use two permanent magnets $(2 \times 20 \mathrm{~cm})$ with a changing distance between the poles $\left(B_{\max }=0.08 \mathrm{~T}\right)$. The charged deuterons are deflected by the magnetic and electric fields. The Ni foil and the polarimeter target are placed in the oppositely directed magnetic fields.

The magnetic field is directed along the foil plane (vertically), and we must use Sona transitions with vertical magnetic fields. This is different from the standard configuration.

The single-crystal nickel foils with a thickness up to $2 \mu \mathrm{m}$ are grown epitaxially on $\mathrm{NaCl}$ crystals cleaved to expose the (110) plane (produced by Princeton Scientific Corp.). The substrate was dissolved by water and the $\mathrm{Ni}$ foils were floated on the $\mathrm{Cu}$ disc mounted on the goniometer.

Vacuum of better than $10^{-4} \mathrm{~Pa}$ was used. 


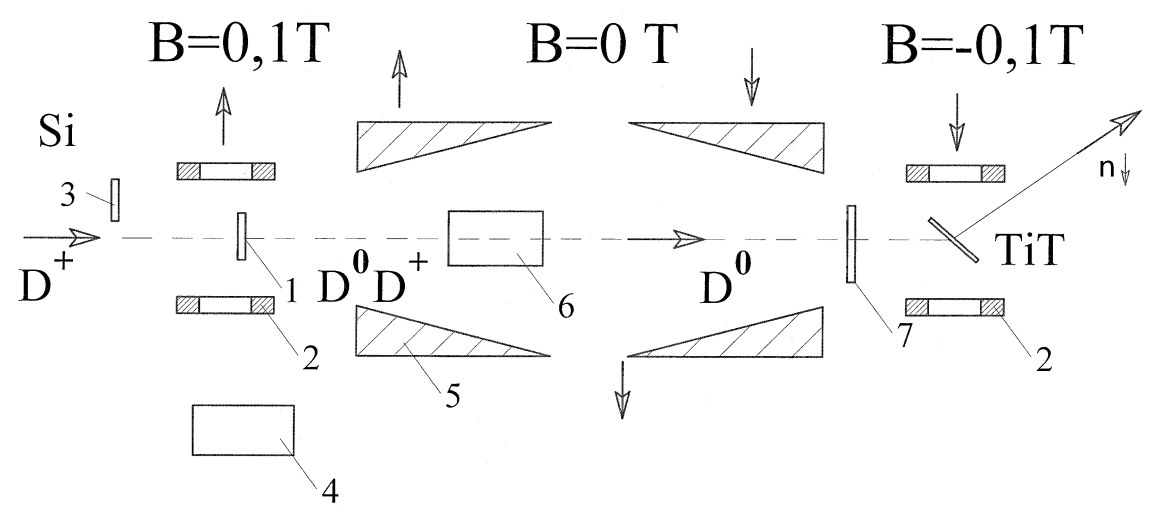

Figure 1: Scheme of the polarized deuteron source; 1 - nickel foil, 2 - permanent magnet $(0.07 \mathrm{~T}), 3$ - solid state detector, 4 - goniometer, 5 - polarizing permanent magnets (for the Sona transitions), 6 - electrostatic plates, 7 - polarimeter target

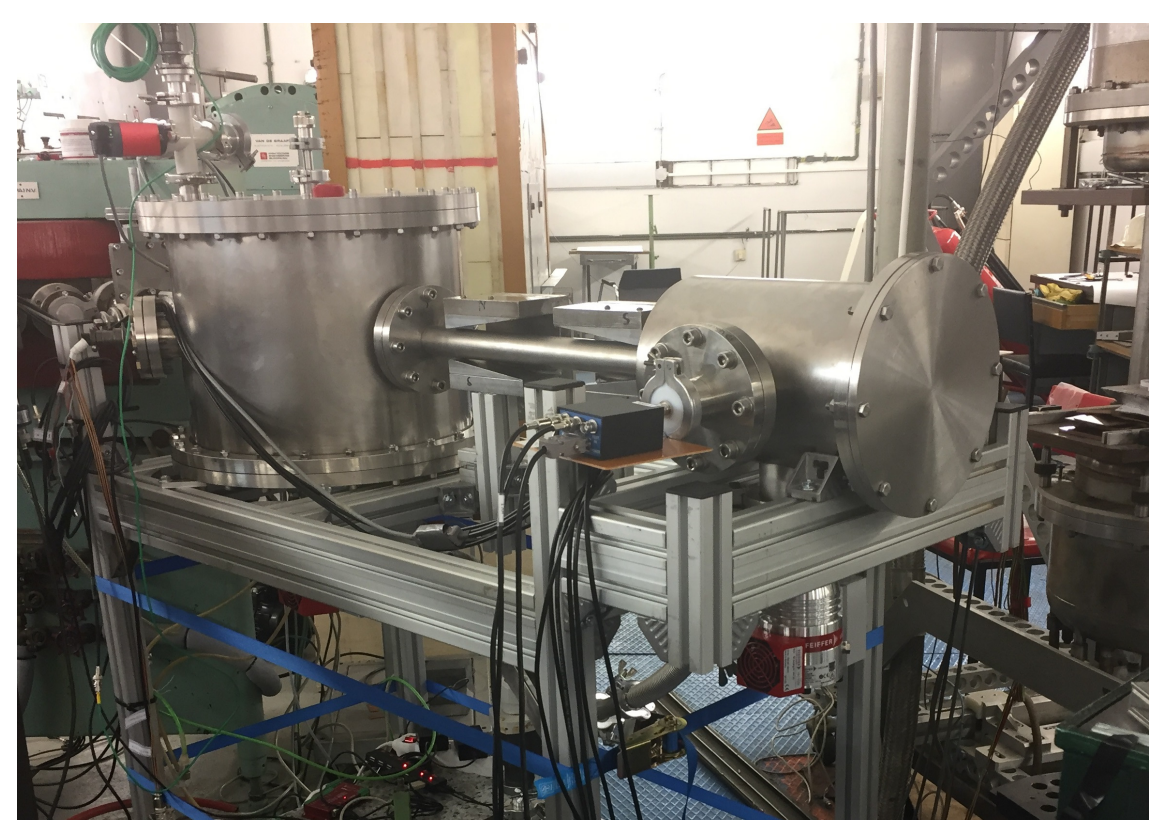

Figure 2: Photo of the experimental setup

The atomic beam in a strong magnetic field has vector polarization of deuterons up to the theoretical limit $P_{3}=2 / 3$ and zero tensor polarization.

If we pass the deuterium beam through a tritium target, $14-\mathrm{MeV}$ neutrons of the $d t$ reaction produced at an angle of $90^{\circ}$ (center of mass) have almost the same vector polarization as the deuterons [21].

\section{Polarimetry}

The deuteron vector polarization may be measured using the reaction $\mathrm{D}(d, p) \mathrm{T}$ [22]. The polarimeter target consisted of deuterated polyethylene with a thickness of about 2-3 $\mu \mathrm{m}$ on a $\mathrm{Cu}$ 
support. The protons produced in this reaction were detected by two surface barrier detectors, each having an effective area of $20 \mathrm{~mm}^{2}$.

The detectors were arranged symmetrically at $\pm 120^{\circ}$ with respect to the beam axis, and the solid angle was $\approx 1 \mathrm{msr}$. In order to suppress the elastically scattered deuterons, ${ }^{3} \mathrm{H}$ and ${ }^{3} \mathrm{He}$, each detector was masked with a $10-\mu \mathrm{m}$-thick aluminum foil. For deuterons with an energy of $400 \mathrm{keV}$ the expected count rate is $4 \mathrm{sec}^{-1}$ per $1 \mu \mathrm{A}$ of the neutral deuterium atoms on the target. The range in $\mathrm{CD}_{2}$ is $0.4 \mu \mathrm{m}$.

For a vector polarized beam the particle intensities detected by two detectors placed on the right and the left of the beam axis are proportional to the cross sections $\sigma_{R}(\theta)$ and $\sigma_{L} \theta$ ), respectively,

$$
\sigma_{R}(\theta)=\sigma_{0 R}(\theta)\left[1-\frac{3}{2} P_{z} A_{y}(\theta)\right]
$$

and

$$
\sigma_{L}(\theta)=\sigma_{0 L}(\theta)\left[1+\frac{3}{2} P_{z} A_{y}(\theta)\right]
$$

where $A_{y}(\theta)$ is the Cartesian analyzing power for the reaction.

Replacing the cross sections by the corresponding right and left detector intensities, $N_{R}$ and $N_{L}$, for polarized beam and $N_{0 R}, N_{0 L}$ for unpolarized beam, we obtain

$$
\frac{N_{R}(\theta)}{N_{L}(\theta)} \times \frac{N_{0 L}(\theta)}{N_{0 R}(\theta)}=\frac{1-3 / 2 P_{z} A_{y}(\theta)}{1+3 / 2 P_{z} A_{y}(\theta)} .
$$

Designating

$$
\kappa=\frac{N_{R}(\theta)}{N_{L}(\theta)} \times \frac{N_{0 L}(\theta)}{N_{0 R}(\theta)}
$$

one obtains

$$
P_{z}=\frac{1-\kappa}{3 / 2(\kappa+1) A_{y}(\theta)} .
$$

According to the calculations, for the real magnetic field value the tensor polarization after the Sona transition is not zero, $P_{z z} \approx 0.1$. In this case, we use the general formula

$$
\begin{gathered}
\sigma(\theta, \phi)=\left[1+\frac{3}{2} \sin \beta \cos \phi P_{z} A_{y}(\theta)-\cos \beta \sin \beta \sin \phi P_{z z} A_{x z}(\theta)\right. \\
\left.-\frac{1}{4} \sin ^{2} \beta \cos 2 \phi P_{z z} A_{x x-y y}(\theta)+\frac{1}{4}\left(3 \cos ^{2} \beta-1\right) P_{z z} A_{z z}(\theta)\right],
\end{gathered}
$$

According to Ad'yasevich [23], at $300 \mathrm{keV} A_{z z}=A_{x x-y y} \approx 0$, at $400 \mathrm{keV} A_{z z}=-A_{x x-y y}=$ -0.1 , and in this energy range additional terms can be neglected.

The measurements of the deuteron vector polarization are in progress.

The tensor polarization was estimated with a TiT target by measuring the angular distribution of the $\alpha$-particles emitted in the reaction $\mathrm{T}(d, n)^{4} \mathrm{He}$ [24].

The cross section depends on the c.m. angle between the spin and the particle direction

$$
\sigma(\theta)=\sigma_{0}\left[1-\frac{1}{4}\left(3 \cos ^{2} \theta-1\right) P_{z z}\right] .
$$

As a result, $P_{z z}=-0.12 \pm 0.04$ at the deuteron energy of $500 \mathrm{keV}$ for the foil thickness $1.5 \mu \mathrm{m}$ (real energy is $250 \mathrm{keV}$ ).During the first experiments the goniometer was at a random position, and we used only one magnet of the Sona transition system. 


\section{Conclusion}

An experimental setup has been developed to produce the beam of deuterium atoms with energies of $100-400 \mathrm{keV}$ with polarized nuclei and to measure vector and tensor polarizations of deuterons.

The ultimate aim is to produce a highly polarized $14-\mathrm{MeV}$ neutron beam for measuring the neutron-deuteron total cross section differences $\Delta \sigma_{L}(n d)$ and $\Delta \sigma_{T}(n d)$ using the frozen spin polarized deuteron target (as a first experiment).

To improve the parameters of the neutron beam, it is proposed to use the reaction $\mathrm{T}(d, n)^{4} \mathrm{He}$ with polarized deuterons with an energy of $100-150 \mathrm{keV}$.

For a nonchanneled beam (the goniometer in a random position), the tensor polarization measurements were carried out with a TiT target in the reaction $\mathrm{T}(d, n)^{4} \mathrm{He}$. Our result is $P_{z z}=$ $-0.12 \pm 0.04$ for a weak field at the target. Feldman's result is $P_{z z}=-0.14 \pm 0.06$.

\section{Acknowledgments}

Authors thank Ivan Štekl for useful discussions.

We thank the Princeton Scientific Corporation for providing $\mathrm{NaCl}$ monocrystals with evaporated $\mathrm{Ni}$.

The work was supported by the European Regional Development Fund-Project "Van de Graaff Accelerator - a Tunable Source of Monoenergetic Neutrons and Light Ions"

(No. CZ.02.1.01/0.0/0.0/16_013/0001785).

\section{References}

[1] S. Ishikawa, M. Tanifuji, Y. Izeri, Forward scattering amplitudes and contributions of three-nucleon forces in nd elastic scattering, in proceedings of 14th Int. Spin Physics Symp., Osaka, 2000, AIP Conf. Proc. 570 (2001) 724-728.

[2] H. Witala et al., Scaling properties of the longitudinal and transversal asymmetries of the $\vec{n} \vec{d}$ total cross section, Phys. Lett. B. 447 (1999) 216-220.

[3] R.D. Foster et al.,Measurement of the relative longitudinal spin-dependent total cross-section difference in $\vec{n}-\vec{d}$ scattering, Phys. Rev. C. 73 (2006) 034002.

[4] J. Broz et al., Measurement of spin-dependent total cross-section difference $\Delta \sigma_{T}$ in neutron-proton scattering at $16 \mathrm{MeV}$, Z. Phys. A. 354 No. 4. (1996) 401-408.

[5] J. Broz et al., Measurement of spin-dependent total cross-section difference $\Delta \sigma_{L}$ in neutron-proton scattering at $16 \mathrm{MeV}$, Z. Phys. A. 359 No. 1. (1997) 23-25.

[6] I. Wilhelm, P. Murali, Z. Doležal., Production of monoenergetic neutrons from the $T(d, n) \alpha$ reaction with the associated particle method, Nucl. Instr. \& Meth. A. 317 (1992) 553-558.

[7] N.S. Borisov et al., Deuteron frozen-spin-polarized target for $n d$ experiments at the VdG accelerator of Charles University, Nucl. Instr. \& Meth. A. 593 (2008) 177-182.

[8] S.T. Goertz et al., Highest polarizations in deuterated compounds, Nucl. Instr. \& Meth. A. 2004526 (2004) 43-52. 
[9] E.K. Zavoiskii, Concerning a possible method for the polarization of a proton beam, Sov. Phys. JETP. 5 (1957) 338-339.

[10] M. Kaminsky, Polarization of channeled particles, Phys. Rev. Lett. 23 (1969) 819-822;

[11] M. Kaminsky, in proceedings of 3rd Int. Symp. on Polarization Phenomena in Nuclear Reactions, Madison (1970) 803-809.

[12] L.C. Feldman, D.W. Mingay, J.P.F. Sellschop, Another measurement of the polarization of deuterons channeled through thin Ni foils, Radiation Effects 13 (1972) 145-151.

[13] W. Gleich, G. Regenfus, R. Sizmann, Spin polarization of field-emitter electrons from monocrystalline Nickel, Phys. Rev. Lett. 27 No. 16. (1971) 1066-1069.

[14] C. Rau, R. Sizmann, Measurement of predominant electron spin orientation at single crystal surfaces of ferromagnetic Nickel, Phys Lett. A. 43 No. 4 (1973) 317-318.

[15] C. Rau, Electron spin polarization ESP at surfaces of ferromagnetic metals, J. Magnetism and Magnetic Materials 30 (1982) 141-174.

[16] K.-H. Speidel et al., Evidence for spin-polarized electrons of highly stripped Fluorine ions emerging from thin ferromagnetic layers, Phys. Rev. Lett. 61 No. 22 (1988) 2616-2619.

[17] M.E. Ebel, Polarization of channeled deuterons, Phys. Rev. Lett. 24 No. 25 (1970) 1395-1398.

[18] W. Brandt, R. Sizmann, Capture of polarized electrons by deuterons emerging from a magnetized Nickel foil, Phys. Lett. A. 37 No. 9 (1971) 115-116.

[19] S. Kreussler, R. Sizmann, Neutralization of 50 - 230-keV Hydrogen ions which have penetrated Al, $A u, C$, and Cs films, Phys. Rev. B. Phys. Rev. 26 No. 2 (1982) 520-529.

[20] P.G. Sona, A New method proposed to increase polarization in polarized ion sources of $H^{-}$and $D^{-}$, Energia Nucleare 14 No. 5 (1967) 295-299.

[21] G.G. Ohlsen, Polarization transfer and spin correlation experiments in nuclear physics, Rep. Progr. Phys. 35 (1972) 717-801.

[22] A.A. Naqvi, G. Clausnitzer, Measurement of beam polarization using the $D(\vec{d}, p)^{3}$ H reaction, Nucl. Instr. \& Meth. A. 324 (1993) 429-432.

[23] B.P. Ad'yasevich, V.G. Antonenko, V.N. Bragin, Research of the reactions ${ }^{2} H(\vec{d}, p)^{3} H$ and ${ }^{2} H(\vec{d}, n)^{3}$ He with a polarized deuteron beam. Extrapolation of the cross sections into the low energy region, Sov. J. Nucl. Phys. 33 (1981) 313.

[24] A. Galonsky, H.B. Willard, T.A. Welton, S-Wave detector of deuteron polarization and 14-MeV polarized-neutron source, Phys. Rev. Lett. 2 No. 8 (1959) 349-351. 